\title{
Evaluation of quality in an ambulatory surgery center: the perianesthesia care from the patient's viewpoint.
}

\author{
Neiva Lemos J. ${ }^{1}$, Dantas Cardoso Neiva Lemos L. ${ }^{2}$, Fontoura Solla D.J. ${ }^{3}$, Dantas \\ Cardoso Neiva Lemos D. ${ }^{2}$, Pinheiro Módolo N.S. ${ }^{1}$
}

${ }^{1}$ Faculdade de Medicina de Botucatu, UNESP, Dept of Anaesthesiology, Botucatu, Brazil, ${ }^{2 B A H I A N A ~-~}$ School of Medicine and Public Health (Escola Bahiana de Medicina e Saúde Pública - EBMSP), Research and Development Department, Salvador, Brazil, ${ }^{3}$ Hospital das Clínicas da Faculdade de Medicina da Universidade de São Paulo (HC/FMUSP), Dept of Neurosurgery, São Paulo, Brazil

\section{Background and Goal of Study}

Quality in anesthesiology is usually measured by morbidity and mortality. Nonetheless, it has been assessed by the patients' satisfaction at various stages of anesthetic care. As satisfaction is the result of care from the client's perspective, the anesthesiologist must be able to build relationships with patients, provide understandable information, involve them in decisions about their anesthesia, answer their questions and listen to their complaints. This measurement therefore provides a basis to improve care in anesthesiology. This study aimed to evaluate peri-anesthetic care in an ambulatory surgery center based on patient's satisfaction measures.

\section{Materials and Methods}

We used the "Heidelberg Peri-anesthetic Questionnaire" to evaluate satisfaction at various stages of peri-anesthetic care in patients undergoing ambulatory surgery. Responses to each question were ranked from " $1-4$ ", corresponding to totally unsatisfied, unsatisfied, satisfied and totally satisfied. Questions with score below pool average minus one standard deviation (SD) and those with a high internal SD were selected for correlation analysis. The correlation analysis using multivariate logistic regression considered the degree of dissatisfaction with patients' characteristics (age, gender, education degree and ASA physical status), anesthesia (type, time and prior experience) and surgical specialty (gynecological, vascular, dermatological, ophtalmic, orthopedic and urological surgery.)

\section{Results and Discussion}

We evaluated 1,211 patients from both sexes, aged 18 to 65 years.

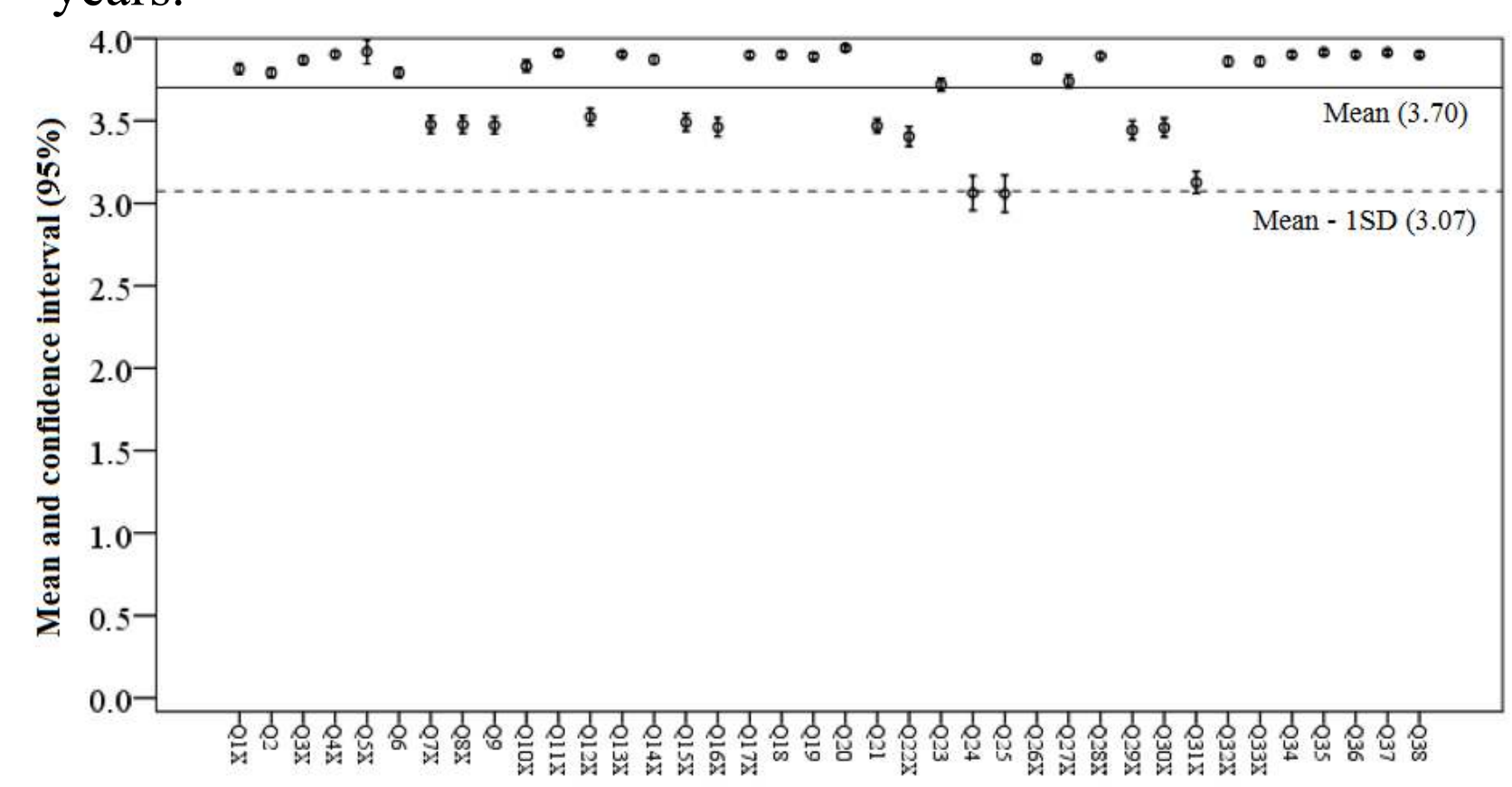

FIGURE 1. Mean and Confidence Interval (95\%) of satisfaction scores for each item. SD: Standard deviation.

References: Schiff JH et al. The Heidelberg Peri-anaesthetic Questionnaire - development of a new refined psychometric questionnaire. Anaesthesia. 2008;63:1096-104.

TABLE 1. Questions related to dissatisfaction

Question

Q7. Fear of anesthesia played an important role

Q8. Fear of surgery played an important role

Q9. The night before surgery felt relaxed

Q12. The waiting time on the day of the surgery was long

Q15. Thirst before the anesthesia was a problem

Q16. Feeling cold or shivering was experienced in the room where the anesthesia was applied

Q21. Waking up from anesthesia was comfortable

Q22. After waking up from anesthesia, pain was experienced in the area of surgery

\section{Q24: Staff members showed they} were seriously concerned about my pain

Q25. The staff quickly alleviated my pain

Q29. Thirst was a problem following anesthesia

Q30. An urgent need to urinate was a problem following anesthesia

Q31. Feeling cold or shivering were problems following the anesthesia.

\section{Variables}

Younger age

Younger age

Younger age

Younger age, dermatological surgery

Younger age, prior anaesthesia (local + sedation)

Younger age, women, college education

Women, gynaecological surgery, prior anaesthesia (local + sedation)

Younger age, women, college education, general anaesthesia, gynecological surgery

Men, prior anaesthesia (general), urological surgery

Men, prior anaesthesia (general), urological surgery

Younger age, college education

Younger age, women, long surgical duration, urological and gynecological surgeries. Younger age, women, long surgical duration.

\section{Conclusion(s)}

The "Heidelberg Peri-anesthetic Questionnaire" proved to be a useful tool in identifying the reasons for patient's dissatisfaction. Younger age, women, college education and general anesthesia were variables related to a greater level of dissatisfaction. Urological and gynecological surgeries and longer surgical duration were also related to dissatisfaction. Feeling cold, thirst, fear and anxiety were related to dissatisfaction. Concern and response speed to postoperative pain management are items that need priority review at the center where this study was developed.

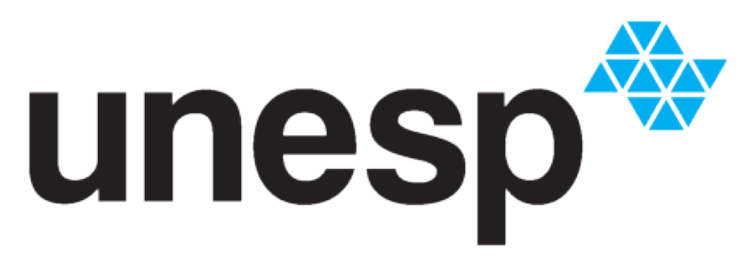

UNIVERSIDADE ESTADUAL PAULISTA "JÚLIO DE MESQUITA FILHO"

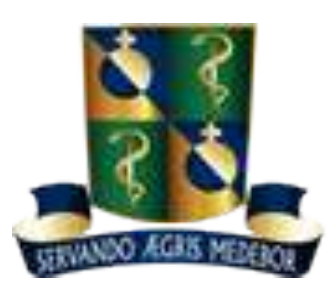

BAHIANA 